\title{
The symmetric six-vertex model and the Segre cubic threefold
}

\author{
M.J. Martins \\ Universidade Federal de São Carlos \\ Departamento de Física \\ C.P. 676, 13565-905, São Carlos (SP), Brazil
}

\begin{abstract}
In this paper we investigate the mathematical properties of the integrability of the symmetric six-vertex model towards the view of Algebraic Geometry. We show that the algebraic variety originated from Baxter's commuting transfer method is birationally isomorphic to a ubiquitous threefold known as Segre cubic primal. This relation makes it possible to present the most generic solution for the Yang-Baxter triple associated to this lattice model. The respective R-matrix and Lax operators are parametrized by three independent affine spectral variables.
\end{abstract}

Keywords: Integrable models, Algebraic Geometry

May 2015 


\section{Introduction}

The six-vertex model is a system of statistical mechanics defined on a square $\mathrm{N} \times \mathrm{N}$ lattice in which the configurations sit on the bonds connecting neighboring lattice points [1]. Its origin goes back to a model introduced by Pauling to explain the experimental fact that the ice crystal has a residual entropy at very low temperatures [2]. The lattice sites are assumed to be occupied by oxygen ions having four nearest hydrogen neighbors. The hydrogen distribution is such that two of them are close to the oxygen ion and the other two are farther away. This means that the sixteen possible states at each lattice vertex are reduced to only six which are shown in Figure 1.

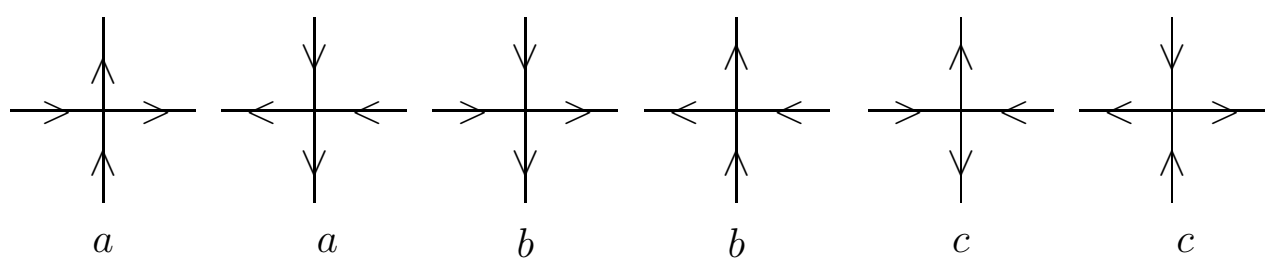

Figure 1: The allowed configurations of the symmetric six-vertex model with the respective energy weights $a, b$ and $c$.

The arrows tip pointing inwards represent states where the hydrogens are close to the oxygen while those outwards represent the hydrogens in more distant positions. In the symmetric case the energy interactions are unchanged by reversing the arrows given rise to only three distinct weights $a, b$ and $c$. It turns out that the model mathematical structure can be captured in terms of a single local operator usually denominated transition or Lax operator. This operator acts on the direct product of the spaces built out of the horizontal and vertical degrees of freedom and its elements are constituted by the energy weights. Since we have two states per bond the Lax operator can be viewed as a two-dimensional matrix whose elements are operators acting on the space $\mathcal{H}=\mathbb{C}^{2 \otimes N}[1,3]$. Its expression in a suitable basis order can be written as,

$$
\mathrm{L}_{j}(a, b, c)=\left(\begin{array}{cc}
a e_{11}^{(j)}+b e_{22}^{(j)} & c e_{21}^{(j)} \\
c e_{12}^{(j)} & b e_{11}^{(j)}+a e_{22}^{(j)}
\end{array}\right), \quad j=1, \cdots, \mathrm{N}
$$


where $e_{l k}^{(j)}$ denote $2 \times 2$ Weyl matrices acting on the subspace $\mathbb{C}_{j}^{2} \in \mathcal{H}$.

In equilibrium statistical mechanics the macroscopic quantities such as the free energy per vertex can be computed after determining the sum $\mathcal{Z}_{\mathrm{N}}$ of the probability distribution. One way to calculate such partition function is to arrange the summations in separated sums over rows of bond state variables. The sum of a single lattice row defines an operator called the model transfer matrix. Assuming periodic boundary conditions the transfer matrix can be written by means of an ordered product of Lax operator as follows [1, 3],

$$
\mathrm{T}(a, b, c)=\operatorname{Tr}_{2}\left[\mathrm{~L}_{\mathrm{N}}(a, b, c) \mathrm{L}_{\mathrm{N}-1}(a, b, c) \cdots \mathrm{L}_{1}(a, b, c)\right],
$$

where the multiplications and the trace operation are performed on the two-dimensional matrix space. We see that the transfer matrix acts on the space $\mathcal{H}$ and in terms of this operator the partition function is given by,

$$
\mathcal{Z}_{\mathrm{N}}=\operatorname{Tr}_{\mathcal{H}}\left[\mathrm{T}(a, b, c)^{\mathrm{N}}\right]
$$

As pointed out by Baxter [4] an important ingredient to determine the dominant large $\mathrm{N}$ behaviour of the partition function is the existence of a family of commuting transfer matrices. This requirement implies the condition,

$$
\left[\mathrm{T}\left(a^{\prime}, b^{\prime}, c^{\prime}\right), \mathrm{T}\left(a^{\prime \prime}, b^{\prime \prime}, c^{\prime \prime}\right)\right]=0
$$

for different set of weights $a^{\prime}, b^{\prime}, c^{\prime}$ and $a^{\prime \prime}, b^{\prime \prime}, c^{\prime \prime}$.

In addition, Baxter argued that a sufficient condition for the above commutation to hold is the existence of an invertible $4 \times 4$ matrix $\check{R}$ satisfying the celebrated Yang-Baxter equation [4],

$$
\check{\mathrm{R}} \mathrm{L}_{j}\left(a^{\prime}, b^{\prime}, c^{\prime}\right) \otimes \mathrm{L}_{j}\left(a^{\prime \prime}, b^{\prime \prime}, c^{\prime \prime}\right)=\mathrm{L}_{j}\left(a^{\prime \prime}, b^{\prime \prime}, c^{\prime \prime}\right) \otimes \mathrm{L}_{j}\left(a^{\prime}, b^{\prime}, c^{\prime}\right) \check{\mathrm{R}},
$$

where the tensor product is taken with respect to the two-dimensional space of the transition operator.

The Yang-Baxter equation consists of a set of algebraic relations on the energy weights and the $\check{\mathrm{R}}$-matrix entries. By eliminating the latter elements one finds the conditions on the Lax operator weights that permit a non-trivial solution to Eq.(51). This approach has been applied for 
the symmetrical six-vertex model [4] and was found that one such solution exists provided the weights are the following subset of the product of two projective spaces,

$$
\mathrm{X}=\left\{\left(a^{\prime}: b^{\prime}: c^{\prime}\right) \times\left(a^{\prime \prime}: b^{\prime \prime}: c^{\prime \prime}\right) \in \mathbb{C P}^{2} \times \mathbb{C P}^{2} \mid \mathrm{F}\left(\left(a^{\prime}, b^{\prime}, c^{\prime} ; a^{\prime \prime}, b^{\prime \prime} c^{\prime \prime}\right)=0\right\}\right.
$$

where the expression of the respective bihomogenous polynomial is,

$$
\mathrm{F}\left(a^{\prime}, b^{\prime}, c^{\prime} ; a^{\prime \prime}, b^{\prime \prime} c^{\prime \prime}\right)=\left[\left(a^{\prime}\right)^{2}+\left(b^{\prime}\right)^{2}-\left(c^{\prime}\right)^{2}\right] a^{\prime \prime} b^{\prime \prime}-\left[\left(a^{\prime \prime}\right)^{2}+\left(b^{\prime \prime}\right)^{2}-\left(c^{\prime \prime}\right)^{2}\right] a^{\prime} b^{\prime} \text {. }
$$

It is not difficult to see that the codimension of the variety $\mathrm{X}$ is one and consequently we are dealing with a three-dimensional algebraic manifold. This threefold contains a two-dimensional subvariety in which the single primed variables are separable from the double primed ones. This particular divisor is formally defined as the product of two identical algebraic sets 1 ,

$$
\mathrm{Y}=\left\{\left(a^{\prime}: b^{\prime}: c^{\prime}\right) \times\left(a^{\prime \prime}: b^{\prime \prime}: c^{\prime \prime}\right) \in \mathbb{C P}^{2} \times \mathbb{C P}^{2} \mid \mathrm{D}\left(a^{\prime}, b^{\prime}, c^{\prime}\right)=0 ; \mathrm{D}\left(a^{\prime \prime}, b^{\prime \prime} c^{\prime \prime}\right)=0\right\}
$$

where the respective polynomial is the quadric,

$$
\mathrm{D}(a, b, c)=a^{2}+b^{2}-c^{2}-\Delta a b, \quad \Delta \in \mathbb{C} .
$$

The above divisor has the appealing feature of providing two independent Lax operators sited on the same algebraic variety. The commutativity of the respective transfer matrices means that their eigenvectors depend on the energy weights only through the parameter $\Delta$. This property has been important for the exact solution of the six-vertex model by Bethe ansatz methods [1, 3].

However, the divisor $\mathrm{Y}$ does not provide us the most arbitrary solution to the Yang-Baxter triple (5) since it is just a subset of X. The understanding of such general solution needs the comprehension of the geometric properties of the original threefold $\mathrm{X}$ which is the aim of the present work. Interesting enough, we shall show that the variety $\mathrm{X}$ is birational to a rather distinguished cubic threefold devised long ago by Segre [5]. In fact, this is the unique cubic threefold having the maximum possible number of ordinary double points as singularities. This connection makes it possible to present the generic solution for the Yang-Baxter triple parametrized in terms of three distinct affine spectral coordinates.

\footnotetext{
${ }^{1}$ Note that the intersection multiplicity of the divisor $\mathrm{Y}$ at $\mathrm{X}$ is one.
} 
We have organized this paper as follows. We start next section reviewing the Baxter approach for the symmetrical six-vertex model providing the means to state our result for the generic solution of the Yang-Baxter triple. In section 3 we elaborate on the demonstration that $\mathrm{X}$ is birationally equivalent to the Segre cubic primal. As byproduct we obtain the parameterization of the single and double prime energy weights.

\section{The Yang-Baxter and main results}

We recall that the Yang-Baxter equation for the six vertex model can be rewritten as a relation acting on the tensor product of three spaces $\mathbb{C}^{2}$. Defining $\check{\mathrm{R}}=\mathrm{PR}$ where $\mathrm{P}$ denotes the transposition on $\mathbb{C}^{2} \otimes \mathbb{C}^{2}$ one finds,

$$
\mathrm{R}_{12} \mathrm{~L}_{13}\left(a^{\prime}, b^{\prime}, c^{\prime}\right) \mathrm{L}_{23}\left(a^{\prime \prime}, b^{\prime \prime}, c^{\prime \prime}\right)=\mathrm{L}_{23}\left(a^{\prime \prime}, b^{\prime \prime}, c^{\prime \prime}\right) \mathrm{L}_{13}\left(a^{\prime}, b^{\prime}, c^{\prime}\right) \mathrm{R}_{12},
$$

The operator $\hat{\mathrm{O}}_{i j} \in \mathrm{C}^{2} \otimes \mathrm{C}^{2} \otimes \mathrm{C}^{2}$ acts as $4 \times 4$ matrix $\hat{\mathrm{O}}$ on the $i$ th and the $j$ th subspaces and as identity on the remaining tensor space component. From Eq.(1) we obtain the matrix representation of the transition operator,

$$
\mathrm{L}(a, b, c)=\left(\begin{array}{cccc}
a & 0 & 0 & 0 \\
0 & b & c & 0 \\
0 & c & b & 0 \\
0 & 0 & 0 & a
\end{array}\right) .
$$

Considering the above Lax operator structure we take an arbitrary R-matrix and substitute both of them on the Yang-Baxter equation (10). These functional relations under the assumption of non-null energy weights fix many elements of the R-matrix to be zero. As a result it is sufficient to choose the R-matrix with the same form of the Lax operator,

$$
R=\left(\begin{array}{cccc}
\mathbf{a} & 0 & 0 & 0 \\
0 & \mathbf{b} & \mathbf{c} & 0 \\
0 & \mathbf{c} & \mathbf{b} & 0 \\
0 & 0 & 0 & \mathbf{a}
\end{array}\right)
$$


where bold letters are used to distinguish R-matrix entries of the energy weights.

Considering the structure of the Lax operator and the R-matrix one finds that the respective Yang-Baxter equation (10) reduces to only three distinct relations [4],

$$
\begin{aligned}
& \mathbf{a} c^{\prime} a^{\prime \prime}-\mathbf{b} c^{\prime} b^{\prime \prime}-\mathbf{c} a^{\prime} c^{\prime \prime}=0, \\
& \mathbf{c} b^{\prime} a^{\prime \prime}-\mathbf{c} a^{\prime} b^{\prime \prime}-\mathbf{b} c^{\prime} c^{\prime \prime}=0, \\
& \mathbf{c} c^{\prime} b^{\prime \prime}+\mathbf{b} a^{\prime} c^{\prime \prime}-\mathbf{a} b^{\prime} c^{\prime \prime}=0 .
\end{aligned}
$$

The vanishing of the above polynomials defines an algebraic variety now on the product of three projective spaces $\mathbb{C P}^{2} \times \mathbb{C P}^{2} \times \mathbb{C P}^{2}$. This algebraic set is strictly speaking reducible since its ideal can be rewritten as the intersection of five distinct primary ideals. This conclusion was reached by studying the ideal primary decomposition with the help of the computer algebra package Singular [6]. However, here we are interested in genuine six-vertex systems in which none of the energy weights vanish everywhere. For instance, we have to disregard the subset of zeros which are sited at the lines $c^{\prime}=0$ or $c^{\prime \prime}=0$ of the projective spaces associated to the Lax operators. It turns out that such restriction is satisfied by only one of the five possible irreducible components. This is equivalent to the condition that the system of homogeneous relations (13,-14) must have non trivial solution for the unknowns $\mathbf{a}, \mathbf{b}$ and $\mathbf{c}$, namely

$$
\left|\begin{array}{ccc}
c^{\prime} a^{\prime \prime} & -c^{\prime} b^{\prime \prime} & -a^{\prime} c^{\prime \prime} \\
0 & -c^{\prime} c^{\prime \prime} & b^{\prime} a^{\prime \prime}-a^{\prime} b^{\prime \prime} \\
-b^{\prime} c^{\prime \prime} & a^{\prime} c^{\prime \prime} & c^{\prime} b^{\prime \prime}
\end{array}\right|=0 .
$$

This determinant becomes proportional to the bihomogenous polynomial (7) defining the threefold X. The polynomials (13-14) can now be solved for the ratios $\mathbf{a}: \mathbf{b}: \mathbf{c}$ and as a result we obtain,

$$
\frac{\mathbf{a}}{\mathbf{c}}=\frac{\left(b^{\prime} a^{\prime \prime}-a^{\prime} b^{\prime \prime}\right) b^{\prime \prime}}{c^{\prime} a^{\prime \prime} c^{\prime \prime}}+\frac{a^{\prime} c^{\prime \prime}}{c^{\prime} a^{\prime \prime}}, \quad \frac{\mathbf{b}}{\mathbf{c}}=\frac{b^{\prime} a^{\prime \prime}-a^{\prime} b^{\prime \prime}}{c^{\prime} c^{\prime \prime}} .
$$

At this point we have introduced the basic ingredients to state our final formulae for the generic solution to the Yang-Baxter triple. Let us denote by the $\mu_{1}, \mu_{2}$ and $\mu_{3}$ the affine spectral variables used to parameterize the algebraic threefold X. We find that Eq.(10) can be rewritten as follows,

$$
\mathrm{R}_{12}\left(\mu_{1}, \mu_{2}, \mu_{3}\right) \mathrm{L}_{13}\left(\mu_{1}, \mu_{2}, \mu_{3}\right) \mathrm{L}_{23}\left(\mu_{3}, \mu_{2}, \mu_{1}\right)=\mathrm{L}_{23}\left(\mu_{3}, \mu_{2}, \mu_{1}\right) \mathrm{L}_{13}\left(\mu_{1}, \mu_{2}, \mu_{3}\right) \mathrm{R}_{12}\left(\mu_{1}, \mu_{2}, \mu_{3}\right) .
$$


It turns out that the matrix structure of the Lax operator normalizing its elements by the energy weight $c$ is,

$$
\mathrm{L}\left(\mu_{1}, \mu_{2}, \mu_{3}\right)=\left(\begin{array}{cccc}
\bar{a}\left(\mu_{1}, \mu_{2}, \mu_{3}\right) & 0 & 0 & 0 \\
0 & \bar{b}\left(\mu_{1}, \mu_{2}, \mu_{3}\right) & 1 & 0 \\
0 & 1 & \bar{b}\left(\mu_{1}, \mu_{2}, \mu_{3}\right) & 0 \\
0 & 0 & 0 & \bar{a}\left(\mu_{1}, \mu_{2}, \mu_{3}\right)
\end{array}\right),
$$

where the expressions for the rational functions $\bar{a}\left(\mu_{1}, \mu_{2}, \mu_{3}\right)$ and $\bar{b}\left(\mu_{1}, \mu_{2}, \mu_{3}\right)$ are given by,

$$
\bar{a}\left(\mu_{1}, \mu_{2}, \mu_{3}\right)=\frac{\mu_{2}-\mu_{1}-\mu_{3}+\mu_{1} \mu_{3}}{\mu_{1}-\mu_{3}-\mu_{1} \mu_{2}+\mu_{1} \mu_{3}}, \quad \bar{b}\left(\mu_{1}, \mu_{2}, \mu_{3}\right)=\frac{\mu_{2}\left(1-\mu_{1}\right)}{\mu_{1}-\mu_{3}-\mu_{1} \mu_{2}+\mu_{1} \mu_{3}},
$$

By the same token the form of the R-matrix can be presented as,

$$
\mathrm{R}\left(\mu_{1}, \mu_{2}, \mu_{3}\right)=\left(\begin{array}{cccc}
\overline{\mathbf{a}}\left(\mu_{1}, \mu_{2}, \mu_{3}\right) & 0 & 0 & 0 \\
0 & \overline{\mathbf{b}}\left(\mu_{1}, \mu_{2}, \mu_{3}\right) & 1 & 0 \\
0 & 1 & \overline{\mathbf{b}}\left(\mu_{1}, \mu_{2}, \mu_{3}\right) & 0 \\
0 & 0 & 0 & \overline{\mathbf{a}}\left(\mu_{1}, \mu_{2}, \mu_{3}\right)
\end{array}\right),
$$

where the non-trivial entries can be computed with the help of Eqs.(17]20). In this sense we observe that Eq.(18) tells us that the double prime energy weights are computed out of Eq.(20) by exchanging $\mu_{1}$ and $\mu_{3}$. Putting these information together and after few simplifications we obtain,

$$
\begin{aligned}
& \overline{\mathbf{a}}\left(\mu_{1}, \mu_{2}, \mu_{3}\right)=\frac{\left(\mu_{1}-\mu_{3}-\mu_{1} \mu_{3}\right)\left(\mu_{3}-\mu_{1}+\mu_{1} \mu_{3}-2 \mu_{2} \mu_{3}+\mu_{2}^{2}\right)}{\left(\mu_{1}-\mu_{3}-\mu_{1} \mu_{2}+\mu_{1} \mu_{3}\right)\left(\mu_{1}-\mu_{3}-\mu_{1} \mu_{3}+\mu_{2} \mu_{3}\right)} \\
& \overline{\mathbf{b}}\left(\mu_{1}, \mu_{2}, \mu_{3}\right)=\frac{\mu_{2}\left(\mu_{3}-\mu_{1}\right)\left(\mu_{1}-\mu_{2}+\mu_{3}-\mu_{1} \mu_{3}\right)}{\left(\mu_{1}-\mu_{3}-\mu_{1} \mu_{2}+\mu_{1} \mu_{3}\right)\left(\mu_{1}-\mu_{3}-\mu_{1} \mu_{3}+\mu_{2} \mu_{3}\right)} .
\end{aligned}
$$

We finally show how the solution associated to the special divisor $\mathrm{Y}$ is obtained as particular case of Eqs.(18,23). We just have to fix the spectral parameters by the relations,

$$
\mu_{1}=\frac{(q-1)\left(t_{1}+t_{2}\right)}{\left(t_{1}+q\right)\left(t_{2}-1\right)}, \mu_{2}=\frac{2 q\left(t_{1}+t_{2}\right)}{\left(t_{1}+q\right)\left(t_{2}+q\right)}, \mu_{3}=\frac{(q-1)\left(t_{1}+t_{2}\right)}{\left(t_{1}-1\right)\left(t_{2}+q\right)},
$$

where $t_{1}$ and $t_{2}$ are free variables related to the affine parameterization of the quadric (9). Here we have also redefined $\Delta=q+1 / q$. 
Considering this constraint in Eqs.(18,-23) we find that the Yang-Baxter equation can be rewritten in the following additive form,

$$
\mathrm{L}_{12}\left(t_{1} / t_{2}\right) \mathrm{L}_{13}\left(t_{1}\right) \mathrm{L}_{23}\left(t_{2}\right)=\mathrm{L}_{23}\left(t_{2}\right) \mathrm{L}_{13}\left(t_{1}\right) \mathrm{L}_{12}\left(t_{1} / t_{2}\right)
$$

where Lax matrix assumes the standard form,

$$
\mathrm{L}(t)=\left(\begin{array}{cccc}
\frac{q^{2}-t^{2}}{t\left(q^{2}-1\right)} & 0 & 0 & 0 \\
0 & \frac{q\left(1-t^{2}\right)}{t\left(q^{2}-1\right)} & 1 & 0 \\
0 & 1 & \frac{q\left(1-t^{2}\right)}{t\left(q^{2}-1\right)} & 0 \\
0 & 0 & 0 & \frac{q^{2}-t^{2}}{t\left(q^{2}-1\right)}
\end{array}\right)
$$

In the context of Algebraic Geometry such particular solution of the Yang-Baxter equation corresponds to an affine algebraic group. In fact, the R-matrix elements are the generators of a map with the group law,

$$
\mathrm{D}\left(a^{\prime}, b^{\prime}, 1\right) \times \mathrm{D}\left(a^{\prime \prime}, b^{\prime \prime}, 1\right) \quad \longrightarrow \quad \mathrm{D}(\mathbf{a}, \mathbf{b}, 1),
$$

since from Eq.(17) we can verify the identity $\mathbf{a}^{2}+\mathbf{b}^{2}-1-\Delta \mathbf{a b}=0$.

\section{Geometric Equivalences}

The Segre cubic threefold $\mathrm{S} \in \mathbb{C P}^{4}$ can be defined by the vanishing of one homogeneous polynomial,

$$
\mathrm{S}=\left\{\left(x_{0}: x_{1}: x_{2}: x_{3}: x_{4}\right) \in \mathbb{C P}^{4} \mid x_{0}^{3}+x_{1}^{3}+x_{2}^{3}+x_{3}^{3}+x_{4}^{3}-\left(x_{0}+x_{1}+x_{2}+x_{3}+x_{4}\right)^{3}=0\right\} \text {. }
$$

It has been known since the end of the $19^{\text {th }}$ century that the hypersurface $\mathrm{S}$ has rather special geometrical properties [5]. This threefold has ten isolated singularities of the simplest type $\mathrm{A}_{1}$ which is the maximum number permitted for a cubic hypersurface in $\mathbb{C P}^{4}$. This property characterizes such hypersurfaces to be the Segre cubic S up to projective equivalences. The basic properties of the Segre cubic primal and its relevance to modern research in Algebraic Geometry have been recently reviewed in [7]. 
In the sequel we shall show that the threefold $\mathrm{X}$ is birationally equivalent to the Segre cubic primal. Here we use the standard result in which the properties of subsets of the product of projective spaces can be investigated in the realm of the theory of plain projective varieties through the Segre embedding, see for example [8]. In particular it follows that X can be realized as the inverse image of some closed set of $\mathbb{C P}^{8}$. More specifically, the Segre map takes a pair of points on $\mathbb{C P}^{2} \times \mathbb{C P}^{2}$ to their pairwise products,

$$
\begin{array}{ccc}
\mathbb{C P}^{2} \times \mathbb{C P}^{2} & \stackrel{\psi}{\longrightarrow} & \mathrm{Q}\left(z_{i j}\right) \subset \mathbb{C P}^{8} \\
\left(a^{\prime}: b^{\prime}: c^{\prime}\right) \times\left(a^{\prime \prime}: b^{\prime \prime}: c^{\prime \prime}\right) & \longmapsto & \left(z_{00}: z_{01}: z_{02}: z_{10}: z_{11}: z_{12}: z_{20}: z_{21}: z_{22}\right),
\end{array}
$$

where the coordinates are identified by the order $z_{00}=a^{\prime} a^{\prime \prime}, z_{01}=a^{\prime} b^{\prime \prime}, \cdots, z_{22}=c^{\prime} c^{\prime \prime}$ and the image $\mathrm{Q}\left(z_{i j}\right)$ is the zero locus of the quadratic equations,

$$
\mathrm{Q}\left(z_{i j}\right)=z_{i j} z_{k l}-z_{i l} z_{k j}, 0 \leq i<k \leq 2 \text { and } 0 \leq j<l \leq 2 .
$$

As the next step to define $\mathrm{X}$ as subvariety of $\mathbb{C P}^{8}$ we rewrite the bihomogenous polynomial (77) in the new variables $z_{i j}$. This is done by paring the single and double prime energy weights according to the Segre map (29) and as a result we obtain the following homogenous polynomial,

$$
\mathrm{F}\left(z_{i j}\right)=z_{00} z_{10}+z_{01} z_{11}-z_{02} z_{12}-z_{00} z_{01}-z_{10} z_{11}+z_{20} z_{21}
$$

The desired system of equations for $\mathrm{X} \in \mathbb{C P}^{8}$ are then obtained by adjoining $\mathrm{F}\left(z_{i j}\right)$ to the quadratic polynomials (30). Thus X can be redefined as follows,

$$
\mathrm{X}=\left\{\left(z_{00}: z_{01}: \cdots: z_{22}\right) \in \mathbb{C P}^{8} \mid \mathrm{F}\left(z_{i j}\right)=\mathrm{Q}\left(z_{i j}\right)=0\right\}
$$

In order to get some insights on the geometric properties of $\mathrm{X}$ we consider this variety on the affine chart $z_{00} \neq 0$. In this open subspace we can use the quadrics $\mathrm{Q}\left(z_{i j}\right)=0$ to eliminate four of the variables such as for example the coordinates $z_{11}, z_{12}, z_{21}$ and $z_{22}$. After this elimination we end up with an affine threefold $\mathrm{T}$ whose projective closure is given by,

$$
\overline{\mathrm{T}}=\left\{\left(z_{00}: z_{01}: z_{02}: z_{10}: z_{20}\right) \in \mathbb{C P}^{4} \mid z_{01}\left(z_{00}^{2}+z_{10}^{2}-z_{20}^{2}\right)-z_{10}\left(z_{00}^{2}+z_{01}^{2}-z_{02}^{2}\right)=0\right\} .
$$


The study of the singular locus of $\overline{\mathrm{T}}$ can again be done with the help of Singular algebra system [6]. We conclude that they are constituted of ten points of type $A_{1}$ and therefore the threefolds $\overline{\mathrm{T}}$ and $\mathrm{S}$ should be projectively equivalent. However, for concrete results, we have to exhibit an explicit example of such linear automorphism which involves a cumbersome combinatorial analysis among projective coordinates. We have been fortunate to find one such possible projective transformation, namely

$$
\left[\begin{array}{l}
x_{0} \\
x_{1} \\
x_{2} \\
x_{3} \\
x_{4}
\end{array}\right]=\left(\begin{array}{ccccc}
1 & 1 & 0 & -1 & 0 \\
0 & -1 & 0 & 0 & 1 \\
0 & -1 & 0 & 0 & -1 \\
0 & 0 & -1 & 1 & 0 \\
0 & 0 & 1 & 1 & 0
\end{array}\right)\left[\begin{array}{l}
z_{00} \\
z_{01} \\
z_{02} \\
z_{10} \\
z_{20}
\end{array}\right] .
$$

At this point we have gathered the basic ingredients to built up morphisms between the therefolds X and the Segre cubic S . Combining the elimination procedure described above with the linear transformation (34) we are able to establish the following rational map,

$$
\begin{array}{ccc}
\mathrm{S} \subset \mathbb{C P}^{4} & \stackrel{\phi}{\longrightarrow} & \mathrm{X} \subset \mathbb{C P}^{8} \\
\left(x_{0}: x_{1}: x_{2}: x_{3}: x_{4}\right) & \longmapsto & \left(\phi_{0}: \phi_{1}: \phi_{2}: \phi_{3}: \frac{\phi_{4}}{\phi_{0}}: \frac{\phi_{5}}{\phi_{0}}: \phi_{6}: \frac{\phi_{7}}{\phi_{0}}: \frac{\phi_{8}}{\phi_{0}}\right),
\end{array}
$$

where the expressions of the polynomials $\phi_{j}$ are,

$$
\begin{aligned}
& \phi_{0}=2 x_{0}+x_{1}+x_{2}+x_{3}+x_{4}, \phi_{1}=-x_{1}-x_{2}, \phi_{2}=x_{4}-x_{3}, \phi_{3}=x_{3}+x_{4}, \phi_{4}=-\left(x_{1}+x_{2}\right)\left(x_{3}+x_{4}\right) \\
& \phi_{5}=-\left(x_{3}-x_{4}\right)\left(x_{3}+x_{4}\right), \phi_{6}=x_{1}-x_{2}, \phi_{7}=-\left(x_{1}-x_{2}\right)\left(x_{1}+x_{2}\right), \phi_{8}=-\left(x_{1}-x_{2}\right)\left(x_{3}-x_{4}\right) .
\end{aligned}
$$

This map is well defined since the closure of the image of $\phi$ is the whole of $\mathrm{X}$. It is also invertible and the respective inverse can the written as,

$$
\begin{array}{ccc}
\mathrm{X} \subset \mathbb{C P}^{8} & \stackrel{\phi^{-1}}{\longrightarrow} & \mathrm{S} \subset \mathbb{C P}^{4} \\
\left(z_{00}: z_{01}: \cdots: z_{22}\right) & \longmapsto & \left(z_{00}+z_{01}-z_{10}: z_{20}-z_{01}:-z_{01}-z_{20}: z_{10}-z_{02}: z_{10}+z_{02}\right),
\end{array}
$$

completing the proof that $\mathrm{X}$ and $\mathrm{S}$ are birational varieties. 


\subsection{Parameterization}

In order to obtain a parameterization for the energy weights it is sufficient to establish a one-toone map from $\overline{\mathrm{T}}$ to $\mathbb{C P}^{3}$. This birational equivalence can be set up exploring the generators of linear system of quadrics passing through five generic points. The technical details of this construction for the Segre cubic primal can, for instance, be found on the pages 149-152 of reference [9]. This method can be adapted to $\overline{\mathrm{T}}$ and in what follows we present only the final result. Let $\lambda_{0}, \cdots, \lambda_{3}$ be the coordinates of $\mathbb{C P}^{3}$ then the desired map is,

$$
\begin{array}{ccc}
\mathbb{C P}^{3} & \stackrel{\varphi}{\longrightarrow} & \overline{\mathrm{T}} \subset \mathbb{C P}^{4} \\
\left(\lambda_{0}: \lambda_{1}: \lambda_{2}: \lambda_{3}\right) & \longmapsto & \left(\varphi_{0}: \varphi_{1}: \varphi_{2}: \varphi_{3}: \varphi_{4}\right),
\end{array}
$$

where the polynomials $\varphi_{j}$ are,

$$
\begin{aligned}
& \varphi_{0}=-\lambda_{0} \lambda_{2}+\lambda_{3}\left(\lambda_{0}-\lambda_{1}+\lambda_{2}\right), \varphi_{1}=\lambda_{1}\left(\lambda_{2}-\lambda_{3}\right), \varphi_{3}=\lambda_{1}\left(\lambda_{0}-\lambda_{3}\right), \\
& \varphi_{2}=\lambda_{2}\left(\lambda_{1}-\lambda_{3}\right)+\lambda_{0}\left(-\lambda_{2}+\lambda_{3}\right), \varphi_{4}=-\lambda_{2} \lambda_{3}+\lambda_{0}\left(-\lambda_{1}+\lambda_{2}+\lambda_{3}\right) .
\end{aligned}
$$

By taking into account the coordinates identification of the Segre embedding (29) we find that the energy weights ratios are given by,

$$
\begin{aligned}
& \frac{a^{\prime}}{c^{\prime}}=\frac{\lambda_{3}\left(\lambda_{0}-\lambda_{1}+\lambda_{2}\right)-\lambda_{0} \lambda_{2}}{\lambda_{0}\left(\lambda_{2}-\lambda_{1}+\lambda_{3}\right)-\lambda_{2} \lambda_{3}}, \frac{b^{\prime}}{c^{\prime}}=\frac{\lambda_{1}\left(\lambda_{0}-\lambda_{3}\right)}{\lambda_{0}\left(\lambda_{2}-\lambda_{1}+\lambda_{3}\right)-\lambda_{2} \lambda_{3}}, \\
& \frac{a^{\prime \prime}}{c^{\prime \prime}}=\frac{\lambda_{3}\left(\lambda_{2}-\lambda_{1}+\lambda_{0}\right)-\lambda_{0} \lambda_{2}}{\lambda_{2}\left(\lambda_{0}-\lambda_{1}+\lambda_{3}\right)-\lambda_{0} \lambda_{3}}, \frac{b^{\prime \prime}}{c^{\prime \prime}}=\frac{\lambda_{1}\left(\lambda_{2}-\lambda_{3}\right)}{\lambda_{2}\left(\lambda_{0}-\lambda_{1}+\lambda_{3}\right)-\lambda_{0} \lambda_{3}} .
\end{aligned}
$$

We now note that the single and double prime ratios are related via the exchange $\lambda_{0} \leftrightarrow \lambda_{2}$. After defining the affine parameters $\mu_{1}=\lambda_{0} / \lambda_{3}, \mu_{2}=\lambda_{1} / \lambda_{3}$ and $\mu_{3}=\lambda_{2} / \lambda_{3}$ we are able to obtain Eqs.(18|20).

\section{Conclusions}

In this paper we have showed that the Baxter's integrability condition for the symmetrical six-vertex model leads us to an algebraic variety which is birationally isomorphic to the Segre 
cubic primal. This threefold has unique geometric properties which are used to provide a parameterization for the R-matrix and Lax operators triple. In general one expects that generic solutions of the Yang-Baxter equation will involve the study of varieties defined as subsets of the

product of two projective spaces. It appears of interest to investigate the geometrical properties of such varieties, with the help of the Segre embedding, for other integrable models of statistical mechanics. This study could reveal whether the ubiquity found here is accidental or may be also present in different ways for other classes of statistical configurations.

\section{Acknowledgments}

This work has been partially supported by the Brazilian Research Agencies FAPESP and CNPq. I am grateful to Daniel Levcovitz and Eduardo Tengan for fruitful discussions on Algebraic Geometry topics over the past years.

\section{References}

[1] E.H. Lieb and F.Y. Wu, Phase Transitions and Critical Phenomena, Vol. 1, eds. C. Domb and M.S. Green, Academic Press, London, 1972

[2] L. Pauling, J. Am. Chem. Soc., 57 (1935) 2680

[3] L.A. Takhtajan and L.D. Faddeev, Usp.Mat.Nauk 34, 34 (1979) 13

[4] R.J. Baxter, Exactly Solved Models in Statistical Mechanics, Academic Press, 1982

[5] C. Segre, Atti.Accad.Sci.Torino, 22 (1887) 791; Mem.Acad.Sci. Torino (2) 39 (1887) 2

[6] W. Decker, G.-W. Greuel and G. Pfisher, Singular 4.0.1, A computer algebra system for polynomial computations, http://www.singular.uni-kl.de, 2014.

[7] I. Dolgachev, Classical Algebraic Geometry: A Modern View, Cambridge University Press, 2012; Corrado Segre and Nodal Cubic Threefolds, arXiv:150106432v1[math.AG]. 
[8] J. Harris, Algebraic Geometry: A First Course, Graduate Texts in Mathematics, Vol. 133, Springer-Verlag, 1992.

[9] H.F Baker, Principles of Geometry, Vol. IV, Cambridge University Press, 1940. 